\title{
Evaluation of Entropy-based Edge Detector Methods for Human Object
}

\author{
Arwa Darwish Alzughaibi \\ -Taibah University \\ Community College \\ Kingdom of Saudi Arabia \\ University of Technology Sydney
}

\author{
Zenon Chaczko \\ University of Technology Sydney \\ Faculty of Engineering and Information Technology
}

\begin{abstract}
In the field of image processing, edges of an image are important as they characterize boundaries. To reduce the volume of data and refine insignificant information without damaging the structural properties of an image, a process called Image Edge Detection may be performed. Understanding algorithms of edge detection is therefore imperative because it is essential in image processing, particularly in object detection. This paper aimed to recognize this importance to detect human object in particular by conducting an experiment, with emphasis on entropy. Similarly, a comparison of the entropy-based edge detector was done based on the different edge detection techniques such as Prewitt, Robert, Sobel, Canny, and LOG operators. Result show that Canny edge detector exhibits a better performance as compared to the other edge detectors to detect the human object in the image. This is derived from the detectors' characteristics including their adaptive nature, sharp edge detection, and performance as applied in noisy image.
\end{abstract}

\section{Keywords}

Edge Detection, Canny, LOG, Soble, Prewitt, Roberts

\section{INTRODUCTION}

In the discipline of image processing and computer vision, the process of detection and extraction of a medium's features is typically done by using a tool called edge detection. In here, digital image points are determined by emphasizing sharp changes. These changes are commonly related to the image's brightness, and through edge detection, the process of finding discontinuities is achieved. In addition, edge detection significantly reduces the amount of data while simultaneously retaining the image's structural properties. This is then used for image processing on a later instance. In essence, contextualization of edges in a grey level image is considered a local feature of the process. The process starts with a separation process through the edge, specifically disconnecting the regions in a neighborhood. This is done to attain a fairly uniform gray level values as seen on both sides of the image. However, edges are not easy to identify in a noisy image for the reason that high frequency contents exist in both the noise and the edge. As a result, this interaction of high frequencies produces an image that is distorted and blurred.

\section{EDGE DETECTION METHODOLOGIES}

In edge detection operation, the most common method used in detecting changes along the gray level gradients is through the application of differential operators [2]. This is categorized into :

(1) First order edge detector, also known as gradient based operator.

(2) Second order edge detector, also known as laplacian based operator.

\subsection{First order edge detector}

This type of edge detector is used along the first order derivative, otherwise referred to as gradient based. As illustrated in the formula below, the image gradient is computed if $I(i, j)$ is considered to be the input image [2].

$$
\nabla(i, j)=i \frac{\partial I(i, j)}{\partial i}+j \frac{\partial I(i, j)}{\partial j}
$$

where:

$i \frac{\partial I(i, j)}{\partial i}$ is the gradient in the i direction

$j \frac{\partial I(i, j)}{\partial j}$ is the gradient in the $\mathrm{j}$ direction.

Gradient magnitude is then computed by using the following formula:

$$
|G|=\sqrt{\left(\frac{\partial I}{\partial i}\right)^{2}+\left(\frac{\partial I}{\partial j}\right)^{2}} \quad \text { or } \quad|G|=\sqrt{G_{i}^{2}+G_{j}^{2}}
$$

therefore:

$$
\theta=\arctan \frac{G_{j}}{G_{i}}
$$

With the calculated gradient magnitude, a stronger edge and a constantly perpendicular gradient direction in relation to the edge's direction are derived.

2.1.1 1. Classical Detector. Under the first order edge detector, the classical operators known as Robert, Sobel, and Prewitt are popular for their relative ease of operation. However, they also show high noise sensitivity. These operators are discussed below. 
Roberts Detector. This type of operator is gradient-based and it functions by computing the sum of the squares derived from the difference between pixels that are diagonally adjacent [6]. This is done through a process called discrete differentiation. This is then followed calculating the image's approximate gradient. Subsequently, the convolution of the input image and the operator's default kernels, as well as the directions and magnitude of the gradient, are calculated through the two $2 \times 2$ kernels as follows:

$$
D_{x}=\left[\begin{array}{cc}
1 & 0 \\
0 & -1
\end{array}\right] \quad \text { and } \quad D_{y}=\left[\begin{array}{cc}
0 & 1 \\
-1 & 0
\end{array}\right]
$$

This operator possesses simplicity, making it advantageous for edge detection. However, this detector is also known to have high noise sensitivity because of the presence of small kernels. This operator also shows incompatibility with today's technology.

Sobel Detector. This operator is commonly applied in computing gradient approximation according to the image intensity function through its discrete differentiation process [6] [5]. . In here, an image's specific pixels are given their corresponding gradient vector, which is referred to as normal to the vector. An entwining is made between the input image and the kernel. This procedure follows a calculation of the direction and magnitude of the gradient by using two $3 \times 3$ kernels as follows:

$$
D_{i}=\left[\begin{array}{lll}
-1 & 0 & +1 \\
-2 & 0 & +2 \\
-1 & 0 & +1
\end{array}\right] \text { and } D_{j}=\left[\begin{array}{ccc}
+1 & +2 & +1 \\
0 & 0 & 0 \\
-1 & -2 & -1
\end{array}\right]
$$

Sobel detector has lower noise sensitivity because of it has bigger kernels when compared with the Robert operator. Because of this, Sobel could compute faster. Accordingly, a reduction of errors as an effect of noise is also seen because of its larger mask. Local averaging is done in this manner as seen in the masks neighborhood [4].

Prewitt Detector. Generally, the Prewitt detector functions almost similar with the Sobel. The main difference, however, lies on the kernels as follows:

$$
D_{i}=\left[\begin{array}{lll}
-1 & 0 & +1 \\
-1 & 0 & +1 \\
-1 & 0 & +1
\end{array}\right] \text { and } D_{j}=\left[\begin{array}{ccc}
+1 & +1 & +1 \\
0 & 0 & 0 \\
-1 & -1 & -1
\end{array}\right]
$$

Because of this particular difference in the kernel, Prewitt operator shows a more superior performance when compared with the Sobel operator [5].

2.1.2 2. Canny edge detector. This detector was developed by John Canny in 1986 [3], has an advanced algorithm that provides a good detection process, a clear response and adaptation, and a good localization. Because of these positive features, Canny is one of the most popular techniques used in image processing. This detector follows four steps in order to attain the maximum effect [9] [2] [8]:

Step1:Noise reduction by smoothing. A convolving of input image $\mathrm{I}(\mathrm{i}, \mathrm{j})$ and Gaussian filter $\mathrm{G}$ is performed in an image with noise leading to a smoother image output. The calculation of the smooth resultant image is derived at:

$$
F(i, j)=G * I(i, j)
$$

step2:Finding gradients. Identification of the edges that show maximum change in the intensity of the grayscale is done by identifying the required areas from the image gradient. It applies Sobel to identify gradient at every pixel in an image that is being smoothened. The formula below corresponds to the Sobel operators in directions $\mathrm{i}$ and $\mathrm{j}$ :

$$
G_{i}=D_{i} * F(i, j) \quad \text { and } \quad G_{j}=D_{j} * F(i, j)
$$

Hence, the magnitude of a pixel's gradient or the edge strength is illustrated as:

$$
G=\sqrt{G_{i}^{2}+G_{j}^{2}}
$$

And the gradient direction is:

$$
\theta=\arctan \left(\frac{G_{j}}{G_{i}}\right)
$$

Therefore, $G_{i}$ and $G_{j}$ represents the gradients illustrated in i- and j-directions, respectively.

step3:Non maximum suppressions. This is done to preserve the gradient images local maxima. Likewise, this also entails the removal of other components to obtain thin edges. Two procedures are followed for pixel M (i, j): First, gradient direction is rounded to the nearest 45 and compared with the pixels gradient magnitude in terms of negative and positive gradient directions. The comparison is based on the gradient direction wherein $E(i, j)$ and $W(i, j)$ are used if the gradient direction is towards the east. In relation, it is also based on the pixels edge strength value and mark. That is, if edge strength of pixel $M(i, j)$ shows a greater value than $E(i, j)$ and $\mathrm{W}(\mathrm{i}, \mathrm{j})$, the gradient value and mark $\mathrm{M}(\mathrm{i}, \mathrm{j})$ are retained as edge pixel. However, if the opposite is the case, then they are either suppressed or removed.

step4:Hysteresis thresholding. During the step called hysteresis thresholding, a single threshold is selected to avoid the issue relating to the streaking, hence, two thresholds $t_{\text {high }}$ and $t_{\text {low }}$ are used. This is done because of the presence of local maxima from noise as part of the non-maxima suppression output.

In order to distinguish pixel as edge, some condition put forth as assumptions particularly for a pixel $M(i, j)$ with gradient magnitude $\mathrm{G}$

(1) If $G<t_{\text {low }}$ then the edge should be discarded.

(2) If $G>t_{\text {high }}$ then the edge is kept.

(3) If $t_{\text {low }}<G<t_{\text {high }}$ and if any of the $3 \times 3$ region neighbors around it possess gradient magnitudes larger than $t_{\text {high }}$, then the edge is kept.

(4) If no neighbors of the pixel( $x, y)$ has high magnitude of gradient but at least a single pixel is in between $t_{\text {low }}$ and $t_{\text {high }}$ then the $5 \times 5$ region is searched to look for any pixel that has amagnitude larger than the $t_{\text {high }}$. Hence, the edge is kept in this situation. Otherwise, the edge is discarded.

\section{2 second order edge detector}

This detector originated from Laplacian $\nabla 2$ which is a second order derivative. This process starts by marking a pixel as edge based on a position that makes an image's second derivative zero [5]. 
The following formula describes the laplacian operator $\nabla 2$ when applied to a $2 \mathrm{D}$ image $\mathrm{I}(\mathrm{i}, \mathrm{j})$ :

$$
\nabla 2=I(i, j)=\frac{\partial^{2}}{\partial x^{2}} I(i, j)+\frac{\partial^{2}}{\partial y^{2}} I(i, j)
$$

2.2.1 Laplacian of Gaussian . . Before the algorithm of Canny became popular, the Marr-Hildreth edge detector was widely known as an edge operator. This edge detector is an operator that is based on gradient and operates with Laplacian with the aim of obtaining an image's second derivative. This is done by operating on the process called zero crossing method. Accordingly, Gaussian and Laplacian operators are used in such procedures. In particular, Gaussian operator is aimed at reducing noise while the Laplacian operator is applied to detect sharp edges [7]

The formula below defines Gaussian function as:

$$
G(i, j)=\frac{1}{\sqrt{2 \Pi \sigma^{2}}} \exp -\left(\frac{i^{2}+j^{2}}{2 \sigma^{2}}\right)
$$

Where, $\sigma$ is standard deviation; while the formula below illustrates the calculation of LOG operator as follows:

$$
\begin{gathered}
L O G=\frac{\partial^{2}}{\partial i^{2}} G(i, j)+\frac{\partial^{2}}{\partial j^{2}} G(i, j)= \\
\frac{i^{2}+j^{2}-2 \sigma^{2}}{\sigma^{4}} \exp \left(-\frac{j^{2}+j^{2}}{2 \sigma^{2}}\right)
\end{gathered}
$$

Despite the advantages of this operator, two major limitations exists:

(1) False edges are seen. These are referred to as responses with no corresponding edges are generated.

(2) A severe localization error is evident when computed through curved edges.

\section{EXPERIMENTATION}

In the process of experimentation, various edge detectors are compared by describing and comparing entropy value comparison. This is done by calculating the entropy of every detector result and identifying the detector that gives the maximum entropy value, which is then selected and considered to be the edge detector that has the most optimum performance, see Table1 each row value represents different values of entropy for a single image using different edge detectors. After the addition of various noise types and different proportions, a process of measurement is done in relation the performance of the different edge detectors. This is then followed by a setting of hierarchy based on the obtained level of performance among the compared edge detectors which is also used as a basis in determining their performance when noise is added.

\subsection{Methodology}

Entropy is chosen as a criteria to evaluate the performance of an edge detector because it helps in measuring the amount of information an edge detector is providing about the image and it is also a differentiating feature which gives different results for each edge detector. Images with human object is used from Graz01 dataset [1] for this experiment to evaluate the performance of various edge detectors to detect the human object.The procedure
Table 1. : Entropy of selected edge detectors is calculated for all the images and these entropy values are compared for the performance measurements

\begin{tabular}{|c|c|c|c|c|}
\hline Sobel & Prewitt & Roberts & LOG & Canny \\
\hline \hline 0.25606 & 0.25565 & 0.18651 & 0.43714 & 0.58571 \\
\hline 0.20160 & 0.20184 & 0.17239 & 0.37494 & 0.45123 \\
\hline 0.24468 & 0.24516 & 0.20162 & 0.38660 & 0.46693 \\
\hline 0.25230 & 0.24941 & 0.21787 & 0.38649 & 0.47852 \\
\hline 0.16442 & 0.16425 & 0.15968 & 0.34221 & 0.47052 \\
\hline
\end{tabular}

starts from the selection of 100 RGB colored images, particularly made in outdoor environments. Subsequently, the obtained images go through a process of conversion from colored image to grayscale ones. The application of the various edge detectors follows through the use of Matlab such as Sobel, Prewitt, Roberts, LOG, and Canny.

\section{RESULT}

Based from the result, as shown in Fig 2 and Fig 1 a , the Canny Edge Detector shows the best performance as compared to the others. This is due to the obvious output demostrating the most number of possible edges and most information, see Fig2 The details of this output are also shown through the plotted entropy values as illustrated in Fig1 a. In here, different colors are used to represent the various plots for each detector.

Findings show that the best performance is seen from the Canny Edge Detector. This is followed by the LOG detector, Sobel, and Prewitt, at an almost equal rate of performance. On the contrary, Roberts detector seem to perform the worst.

The original image is then incorporated with noise before testing the edge detectors. The noises include Gaussian, Salt \& Pepper and Speckle. Similarly,different proportions of these various noises are added for testing with the values: $(0.1,0.2,0.3,0.4,0.5,0.6$, $0.7,0.8,0.9,1)$. As a result, Canny Edge Detector shows the best performance despite the added noises as seen in Fig1 b,c,d).

\section{ADVANTAGES OF CANNY EDGE DETECTION ALGORITHM}

In summary, some advantages and disadvantages relating to the use of Canny edge detection algorithm have been discussed in this paper. Based from the analysis of the different techniques used in edge detection, Canny has been found to give the best edge detection potential. Further discussion on the advantages that contribute to this optimum performance is presented in this section.

(1) Less Sensitive to noise

The Canny operator has a lower noise sensitivity when compared with other edge detectors such as Prewitt, Robert and Sobel. This is because of its Gaussian filter used in removing noise. This filter is seen to be better than what the other operators are using. Similarly, Canny also shows better performance in terms of noise sensitivity, particularly when associated with the higher sensitivity level of the LoG operator.

(2) Remove streaking problem

Canny is also seen to have a better performance with respect to its ability to remove the problem of streaking. As compared to Robert, which utilizes a technique known as single thresholding, Canny fares better because of its hysteresis technique. This is true for the reason that two threshold values are used in the process. In here, streaking refers to the process of removing essential parts of the connected edge when the 


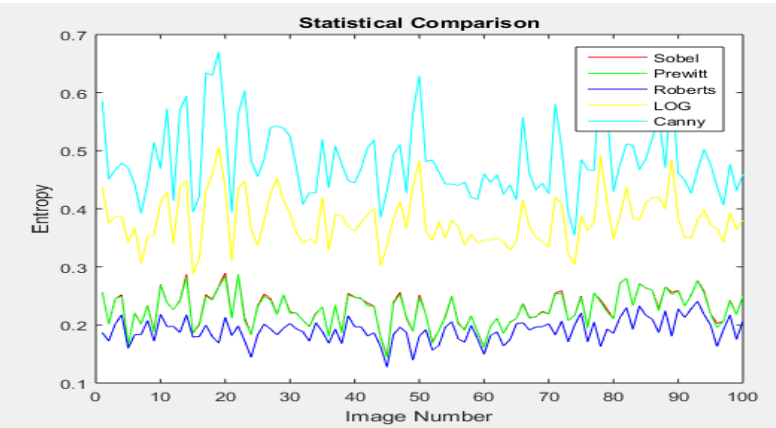

(a)

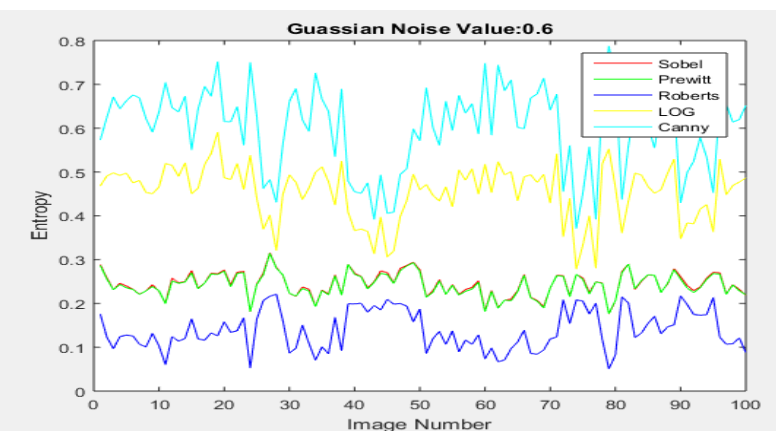

(b)

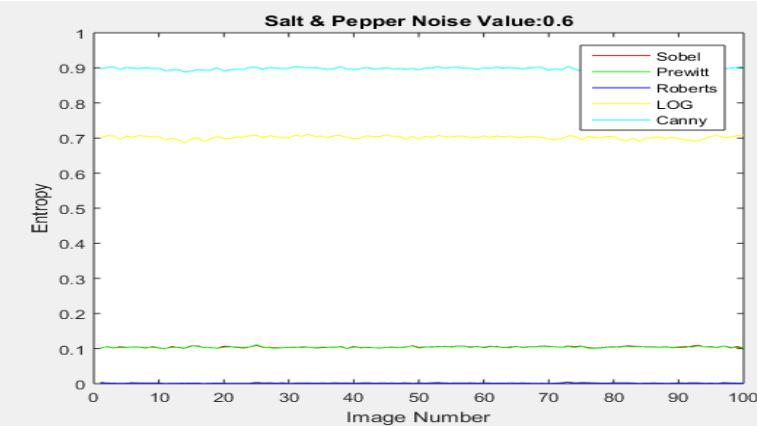

(c)

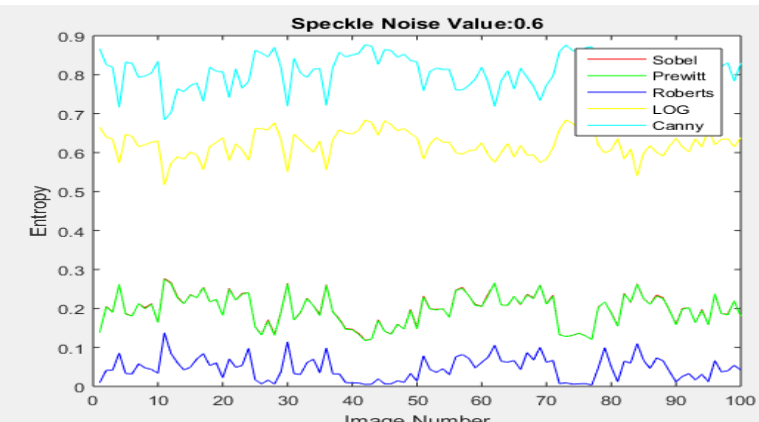

(d)

Fig. 1: Evaluation performance for various edge detectors based on entropy

gradient of the edge is either just below or just above the set threshold limit, thereby leaving the disconnected final edge.

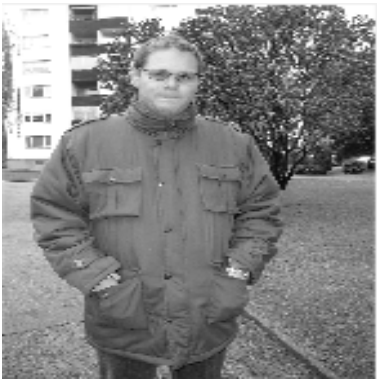

(a) inputimage

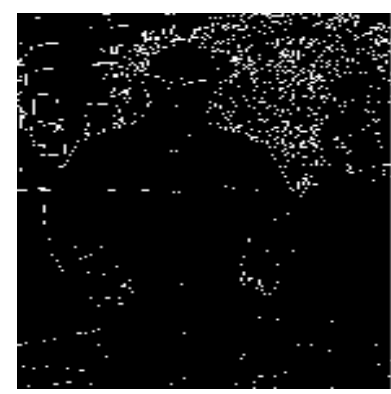

(c) Prewitt

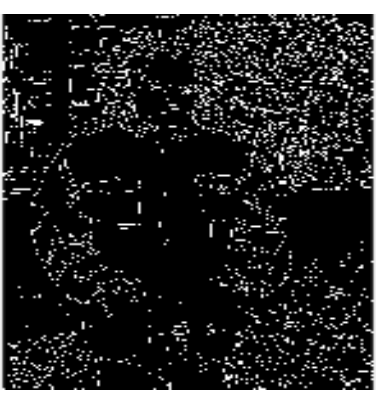

(e) LOG

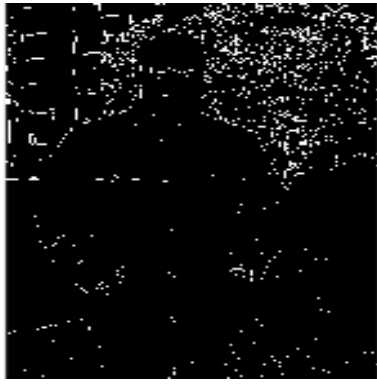

(b) Soble

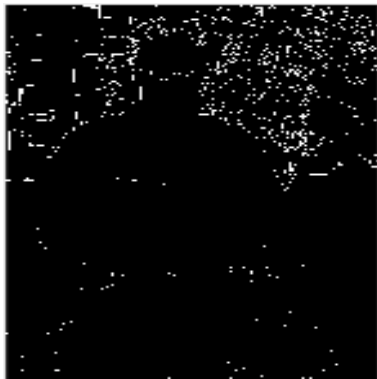

(d) Robert

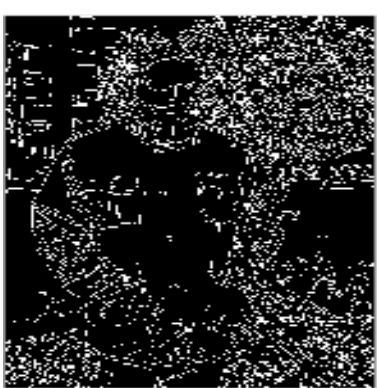

(f) Canny
Fig. 2: The output of the various edge detectors

(3) Adaptive in nature

Canny allows the user to change parameters such as Gaussian filter and threshold values to obtain finer edges. Because of the presence of fixed kernels in classical operators which could not be adapted based on a certain Image, this parameters could not be adjusted when other operators are used. The canny algorithm is advantageous because it depends on these variables or the adjustable parameters such as $\sigma$ (Gaussian filter standard deviation) and the threshold values $t_{\text {low }}$ and $t_{\text {high }}$. With this, the canny algorithm result is also improved in the process.

(4) Good localization

Lastly, Canny operator offers an orientation process involving edge gradient. This process contributes to the derivation of good localization as compared to LoG operators that are encountering difficulty in determining edge orientation. 


\section{CONCLUSION}

This paper evaluated the various edge detection techniques that are most often used in Gradient-based and Laplacian-based Edge Detection to detect the human object in the image. As a result of experimentation, the paper posits that Canny edge detector provides better and more optimized performance when compared with other operators. This is based on its characteristics that show low sensitivity to noise, being more adaptive, better performance in resolving streaking problem, better localization, and better way of detecting sharper edges. Thus, this operator is considered as the best technique for edge detection. Nonetheless, a lot of potential is still untapped from this operator and these are needed to be explored further. Additionally, its algorithm is still to be improved in order to attain an unmatched performance for the best possible edge detection

\section{REFERENCES}

[1] Graz01 dataset object detection. http://www .emt.tugraz. at/ pinz/data/GRAZ_01/ Accessed: 2015-09-30.

[2] Mrs Anandhi, MS Josephine, V Jeyabalaraja, and S Satthiyaraj. Comparison of canny and sobel edge in detection techniques. INTERNATIONAL JOURNAL OF ENGINEERING SCIENCES \& RESEARCH TECHNOLOGY, 4:550-555, 2015.

[3] John Canny. A computational approach to edge detection. Pattern Analysis and Machine Intelligence, IEEE Transactions on, (6):679-698, 1986.

[4] Binsu C Kovoor, MH Supriya, and K Poulose Jacob. Effectiveness of feature detection operators on the performance of iris biometric recognition system. International Journal of Network Security \& Its Applications (IJNSA), 5(5), 2013.

[5] Raman Maini and Himanshu Aggarwal. Study and comparison of various image edge detection techniques. International journal of image processing (IJIP), 3(1):1-11, 2009.

[6] N Senthilkumaran and R Rajesh. Edge detection techniques for image segmentation-a survey of soft computing approaches. International journal of recent trends in engineering, 1(2), 2009.

[7] GE Sotak and Kim L Boyer. The laplacian-of-gaussian kernel: a formal analysis and design procedure for fast, accurate convolution and full-frame output. Computer Vision, Graphics, and Image Processing, 48(2):147-189, 1989.

[8] Jianjun Zhao, Heng Yu, Xiaoguang Gu, and Sheng Wang. The edge detection of river model based on self-adaptive canny algorithm and connected domain segmentation. In Intelligent Control and Automation (WCICA), 2010 8th World Congress on, pages 1333-1336. IEEE, 2010.

[9] Peng Zhao-Yi, Zhu Yan-Hui, and Zhou Yu. Real-time facial expression recognition based on adaptive canny operator edge detection. In Multimedia and Information Technology (MMIT), 2010 Second International Conference on, volume 2, pages 154-157. IEEE, 2010. 